\title{
Wearable Wireless Temperature Sensor Nodes Appressed to Base of a Calf's Tail
}

\author{
Hirofumi Nogami ${ }^{1,2, *}$, Hironao Okada ${ }^{2}$, Toru Miyamoto ${ }^{3}$, \\ Ryutaro Maeda ${ }^{2}$ and Toshihiro Itoh ${ }^{2}$ \\ 'Department of Mechanical Engineering, Faculty of Engineering, Kyushu University, \\ 744 Motooka, Fukuoka, Fukuoka 819-0395, Japan \\ ${ }^{2}$ National Institute of Advanced Industrial Science and Technology (AIST), \\ 1-2-1 Namiki, Tsukuba, Ibaraki 305-8564, Japan \\ ${ }^{3}$ National Institute of Animal Health, \\ 3-1-5 Kannondai, Tsukuba, Ibaraki 305-0856, Japan
}

(Received April 23, 2014; accepted September 5, 2014)

Key words: wearable wireless sensor node, animal health monitoring, body temperature

Respiratory diseases in calves are the primary cause of infantile death since calves have low resistance to viruses or bacteria and are vulnerable to respiratory diseases such as pneumonia. An effective method used successfully for the early detection of respiratory diseases is to measure the rectal temperature of a calf using a thermometer. However, this method can only be conducted infrequently since it requires significant time and effort from farmers during group feeding. In order to minimize the time and effort required, we developed wearable wireless sensor nodes to automatically measure the body temperature of a calf. In our previous study, we succeeded in measuring the body temperature via wireless sensor nodes attached to a calf's tail, and correlated it with the rectal temperature. However, the wireless sensor nodes developed in that study would often indicate a lower temperature. The cause was due to a gap, which was attributed to the $7 \mathrm{~mm}$ thickness of the sensor nodes, between the measurement location on the calf and the temperature sensor. In order to address these problems, we designed new sensor nodes that were best suited to measure the temperature of the base of a calf's tail. As a result, we could accomplish measurement stability for the temperature sensor.

\section{Introduction}

To achieve the goal of creating a safer and more secure society, wireless sensor network technology has been a promising approach for a variety of applications, such as structural health monitoring, human body monitoring, and animal health monitoring. ${ }^{(1-6)}$ Structural health monitoring is used to improve the safety and reliability of buildings,

*Corresponding author: e-mail: hirofumi-nogami@aist.go.jp 
bridges, and lifeline systems by detecting damage before it reaches a critical state. This damage is detected through many wireless sensor nodes installed in the structure. ${ }^{(1,2)}$ Human body monitoring detects sleep disorders, Parkinson's disease, and other diseases by recording a person's daily movement and posture using a GPS and triaxial and magnetic sensors. ${ }^{(3)}$ These technologies have also been introduced in agriculture in areas such as animal health monitoring. ${ }^{(4-7)}$ It is considered that wireless sensor nodes attached to animals, in conjunction with a wireless health monitoring system, can achieve early detection and prevention of diseases, and therefore minimize economic losses. ${ }^{(8,9)}$ In this work, we focused on the development of wearable wireless sensor nodes for applications in calf health monitoring.

Calves are less able to fight respiratory diseases such as pneumonia, and the rates of death and disease caused by respiratory illnesses are the highest among all types of incident. To prevent these incidents, the accurate measurement of the rectal temperature of calves using a thermometer is necessary to detect fever. However, this method requires significant time and effort from the farmer. As an alternative, we have developed an animal health monitoring system which automatically detects sick calves (Fig. 1). In this system, each calf wears a wireless sensor node. The sensor node measures the temperature and detects the presence of fever in a calf. We have developed wearable wireless sensor nodes for this system.

In a previous work, we could measure the skin temperature for 16 days [Fig. 2(a)]. ${ }^{(10)}$ In this graph, we attached compact wireless sensor nodes [Fig. 2(b)] to the base of a calf's tail [Fig. 2(c)]. The sensor nodes, which can measure both temperature and acceleration, are $18 \times 18 \times 7 \mathrm{~mm}^{3}$ and weigh $6.6 \mathrm{~g}$ with batteries. Their primary advantage is their compactness and small size, allowing easy attachment to any part of the calf. The relationship between the daily maximum rectal temperature and the daily

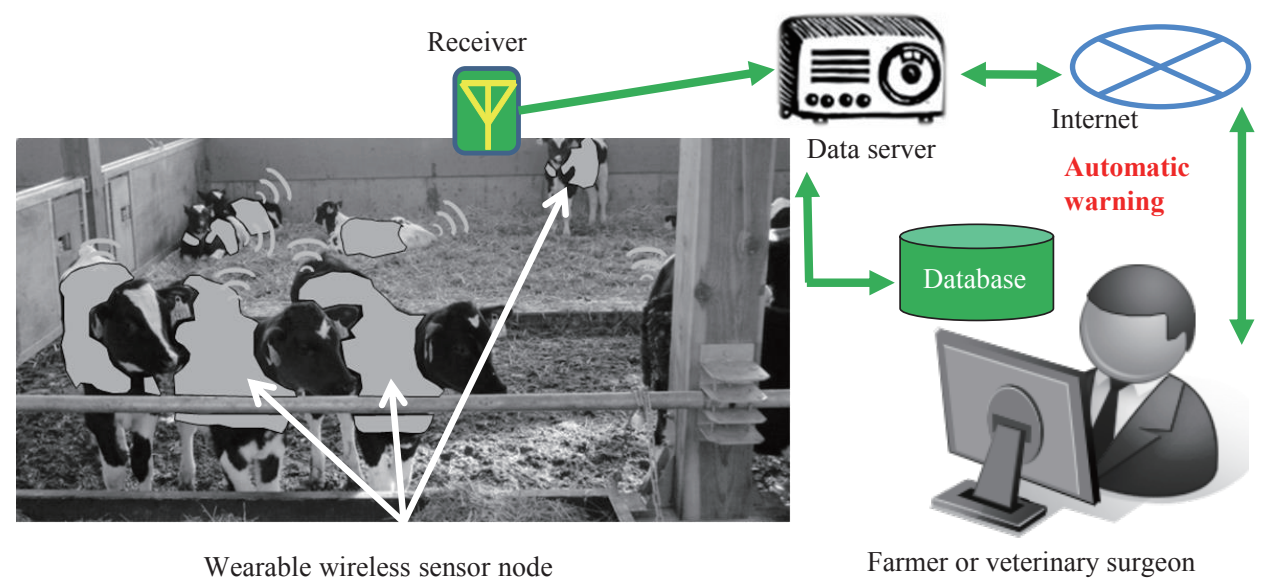

Fig. 1. (Color online) Calf health monitoring system. 


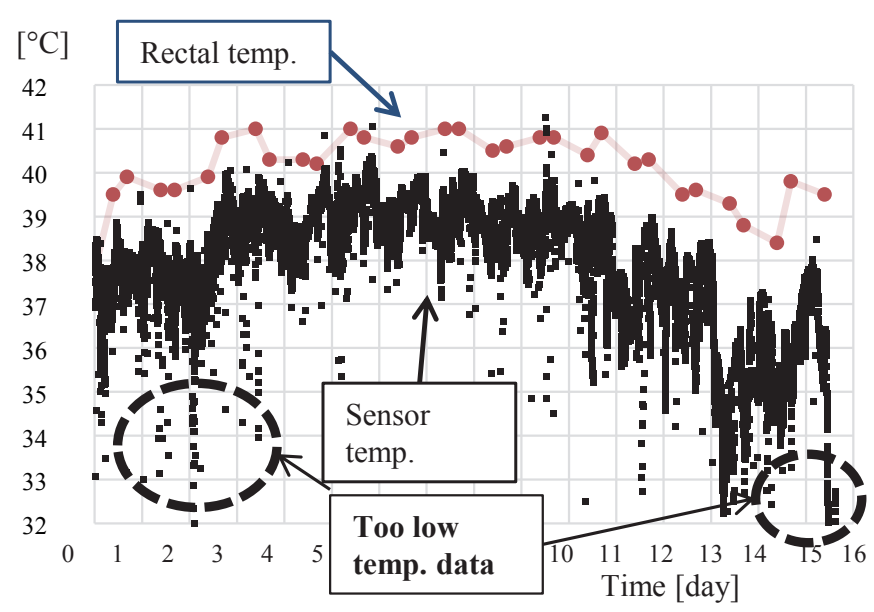

(a)

Size

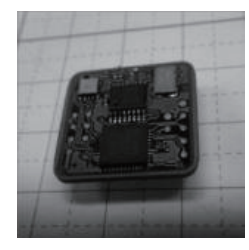

(b)
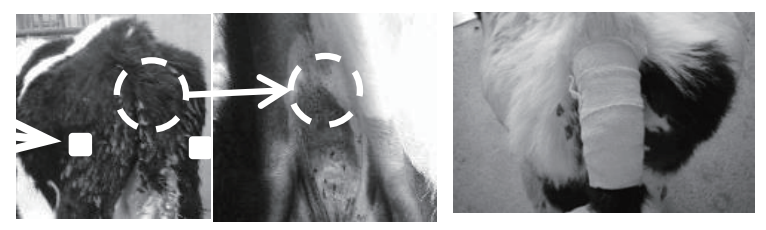

(c)

Fig. 2. (Color online) Early studies: (a) measurement results of continuous monitoring over 16 days, (b) old wireless sensor node, and (c) attachment position.

maximum sensor temperature exhibited a strong correlation. These results revealed that wearable wireless sensor nodes can detect calf fever by continuously measuring the skin temperature instead of measuring the rectal temperature.

However, the wireless sensor nodes indicated a temperature that was very low in some cases, and the frequency of this low estimation increased over time [dashed circle in Fig. 2(a)]. The cause was considered to be the gap between the skin of the calf and the temperature sensor part of the wireless sensor node. The gap was caused by the $7 \mathrm{~mm}$ thickness of the sensor nodes.

To address these problems, we designed new wireless sensor nodes that were best suited to measure the skin temperature of a calf's tail. The temperature unit consists of a thermistor and a flexible substrate. Thus, the temperature unit can be bent to attach it to a curved surface such as a calf's tail. We examined the accuracy of temperature sensor measurement. 


\section{Materials and Methods}

\subsection{Design of the wireless sensor nodes}

We designed wireless sensor nodes that can be appressed to the base of a calf's tail and which can easily loop around it. The sensor nodes were divided into two units. One unit was the temperature unit, and the other was the main unit. The temperature unit had an integrated circuit temperature sensor mounted on a flexible substrate so that the temperature unit could be bent to attach it to a curved surface. The temperature unit can be appressed to the base of the calf's tail.

The main unit contains the data processing function, the data transmission function, and the battery. The data processing function used an F930 (Silicon Labs, Inc.) and the data transmission function used a TRC103 (RF Monolithics, Inc.). The battery was a $3 \mathrm{~V}$ CR2032.

Figures 3(a) and 3(b) show photographs and a schematic diagram of the sensor nodes, respectively. The temperature unit is on the right, and the main unit is on the left in Fig. 3(a). The size of the main unit was $25.3 \times 25.3 \times 8 \mathrm{~mm}^{3}$ and the size of the temperature unit was $5.0 \times 4.0 \times 0.8 \mathrm{~mm}^{3}$ [dashed circle in Fig. 3(a)]. The weight of the sensor node was $2.9 \mathrm{~g}$ without the battery. In this work, the battery was a CR2032 (3.0 g), and the total weight of the sensor node was therefore $5.9 \mathrm{~g}$.

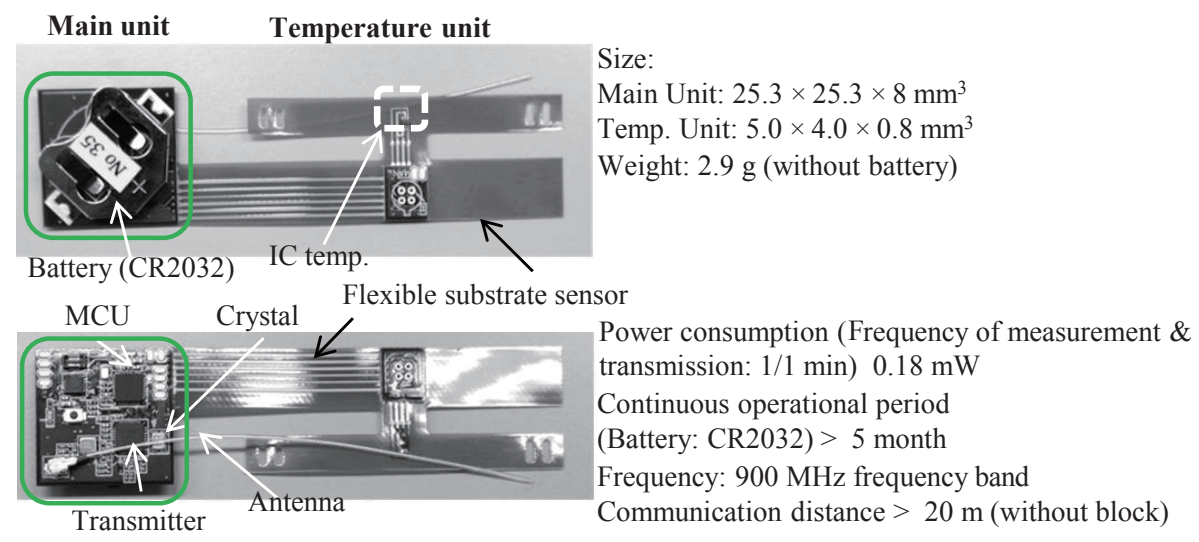

(a)

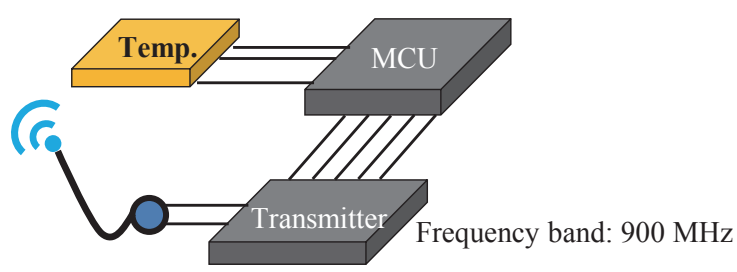

Wire antenna

(b)

Fig. 3. (Color online) Fabricated wireless sensor node: (a) photograph and (b) block diagram. 
The operating frequency of the transceiver was chosen to be $900 \mathrm{MHz}$ because the permeability of radio waves at this frequency is high for humans and animals. ${ }^{(11)}$ The communication distance was approximately $20 \mathrm{~m}$ without obstacles. The measurement and transmission interval was set to $1 \mathrm{~min}$, giving a power consumption of $0.18 \mathrm{~mW}$. The lifetime of the sensor nodes was longer than 5 months using the CR2032 battery.

\subsection{Experimental procedure}

In Fig. 4, photographs of the evaluation experiment are shown. The experimental object is a Holstein calf [Fig. 4(a)], which has the possibility of contracting pneumonia. The calf was 3 months old. The measurement was taken at the base of the tail. The temperature unit of the sensor node was fixed on a sponge as shown in Fig. 4(b). We could easily attach the sensor nodes to the base of a calf's tail using a belt [Fig. 4(c)].

To confirm the measurement stability, we measured the temperature of the base of a calf's tail. Measurements were performed continuously on three calves over a period of 5 days.

\section{Results and Discussion}

Figure 5 shows the measurement results of the three calves for the 5 day period. The measurement temperature is shown by the lower line and the maximum temperature every $30 \mathrm{~min}$ is shown by the upper line. The sensor temperature of less than $33{ }^{\circ} \mathrm{C}$ was not observed. Also, the variability of the anteroposterior data was low. In a previous work, ${ }^{(10)}$ the temperature data often indicated values below $33{ }^{\circ} \mathrm{C}$, and the anteroposterior data varied widely. From these results, it can be seen that additional measurement stability is provided by the thinness of the temperature unit $(0.8 \mathrm{~mm})$.

As a result of the additional measurement stability, the circadian temperature rhythm, which varied between 34 and $38{ }^{\circ} \mathrm{C}$, could be clearly identified. Additionally, it is advisable to determine the correlation between the rectal temperature and the sensor temperature, which is computed as the maximum temperature data every $30 \mathrm{~min}$ (upper line in Fig 5). It can be seen that the sensor temperature can be used to detect fever, similarly to the measured rectal temperature.

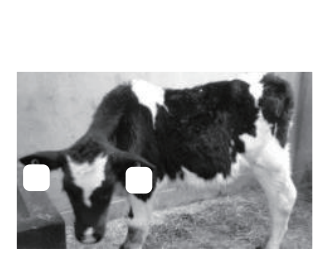

(a)

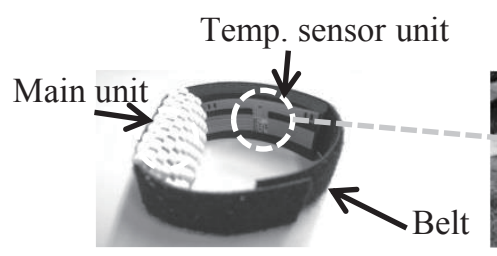

(b)

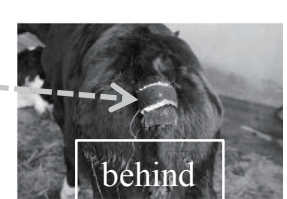

(c)

Fig. 4. Measurement setup: (a) target, (b) new wireless sensor node, and (c) attachment position. 
Target 1
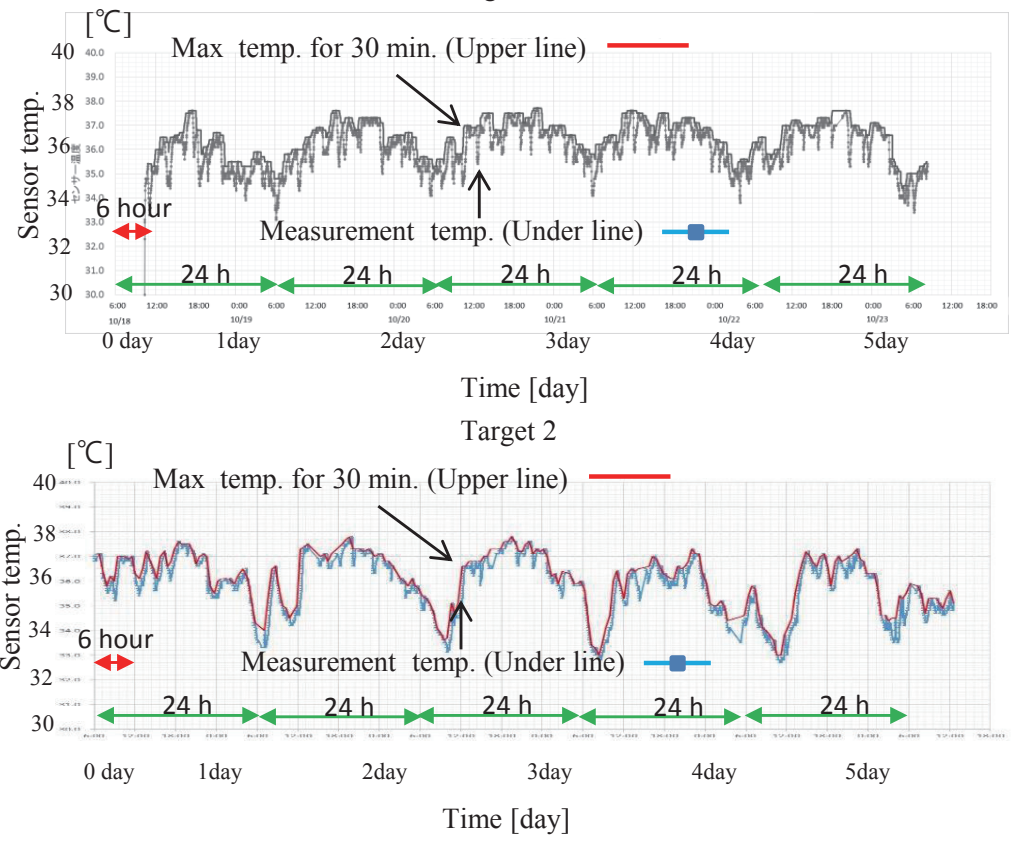

$\left[{ }^{\circ} \mathrm{C}\right]$

Target 3

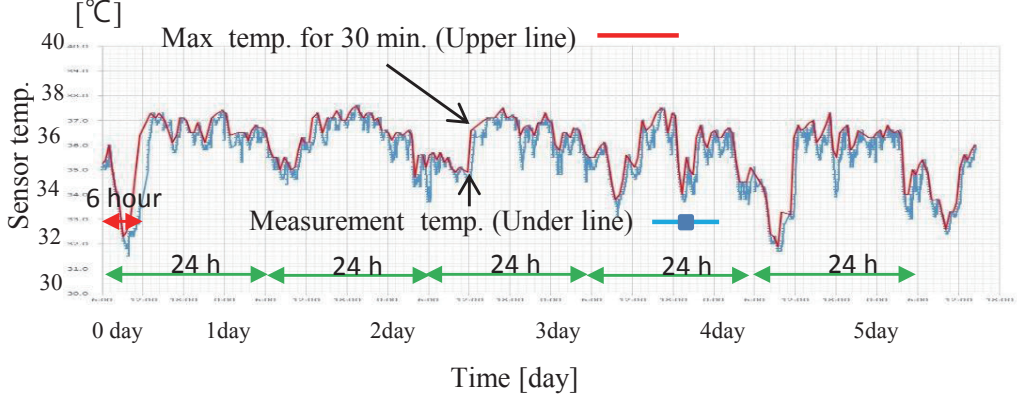

Fig. 5. (Color online) Measurement results of three calves for the 5 day period.

\section{Conclusions}

In this paper, we described newly designed wireless sensor nodes that are best suited to measure the skin temperature at the base of a calf's tail. The sensor nodes consist of a temperature unit and a main unit. Since the temperature unit had an integrated circuit temperature sensor mounted on a flexible substrate, we could appress the temperature unit to the base of a calf's tail. In addition, this belt-type sensor can be easily mounted and removed and is considered suitable for practical use. 
The measurement results were observed to be very stable. We could clearly confirm the circadian temperature rhythm because the new sensor nodes did not measure dropped data. Thus, the sensor nodes can detect an initial increase in temperature, and detect infected calves early. Using the sensor nodes, in the future, we intend to confirm measurements taken from infected calves.

\section{Acknowledgement}

This work was supported by Grant-in-Aid for Scientific Research (A) No. 24248044.

\section{References}

1 S. G. Taylor, K. M. Farinholt, E. B. Flynn, E. Figueiredo, D. L. Mascarenas, E. A. Moro, G. P. Park, M. D. Todd and C. R. Farrar: Meas. Sci. Technol. 20 (2009) 045201.

2 J. A. Rice, K. Mechitov, S. H. Sim, T. Nagayama, S. Jang, R. Kim, B. F. Spencer Jr., G. Agha and Y. Fujino: Smart Struct. Syst. 6 (2010) 423.

3 A. Olivares, G. Olivares, F. Mula, J. M. Górriz and J. Ramírez: J. Syst. Archit. 57 (2011) 905.

4 S. E. Díaz, J. C. Pérez, A. C. Mateos, M. C. Marinescu and B. B. Guerra: Comput. Electron. Agric. 76 (2011) 252.

5 Y. Guo, P. Corke, G. Poulton, T. Wark, G. Bishop-Hurley and D. Swain: Proc. IEEE Local Computer Networks, 2011, p. 607.

6 P. Sikka, P. Corke and L. Overs: Proc. IEEE Local Computer Networks, 2004, p. 446.

7 K. Nishihara, W. Iwasaki, M. Nakamura, E. Higurashi, T. Soh, T. Itoh, H. Okada, R. Maeda and R. Sawada: IEEE Trans. Biomed. 60 (2013) 1645.

8 H. Okada, T. Itoh, K. Suzuki, T. Tatsuya and K. Tsukamoto: Proc. 9th Conf. Sensors, 2010, p. 660 .

9 H. Okada, H. Nogami, T. Itoh and T. Masuda: Proc. dMEMS2012 Conf. Design, Control and Software Implementation for Distributed MEMS (dMEMS), 2012, p. 90.

10 H. Nogami, H. Okada, T. Miyamoto, R. Maeda and T. Itoh: Sens. Mater. 25 (2013) 577.

11 A. Lerchl: Prog. Biophys. Mol. Biol. 107 (2011) 404. 